This item was submitted to Loughborough's Research Repository by the author.

Items in Figshare are protected by copyright, with all rights reserved, unless otherwise indicated.

\title{
Quantifying non-Markovianity due to driving and a finite-size environment in an open quantum system
}

\section{PLEASE CITE THE PUBLISHED VERSION}

https://doi.org/10.1103/PhysRevA.95.022120

\section{PUBLISHER}

(C) American Physical Society

\section{VERSION}

VoR (Version of Record)

\section{PUBLISHER STATEMENT}

This work is made available according to the conditions of the Creative Commons Attribution-NonCommercialNoDerivatives 4.0 International (CC BY-NC-ND 4.0) licence. Full details of this licence are available at: https://creativecommons.org/licenses/by-nc-nd/4.0/

\section{LICENCE}

CC BY-NC-ND 4.0

\section{REPOSITORY RECORD}

Sampaio, Rui, Samu Suomela, Rebecca Schmidt, and Tapio Ala-Nissila. 2019. "Quantifying Non-markovianity Due to Driving and a Finite-size Environment in an Open Quantum System". figshare. https://hdl.handle.net/2134/28171. 


\title{
Quantifying non-Markovianity due to driving and a finite-size environment in an open quantum system
}

\author{
Rui Sampaio, ${ }^{1, *}$ Samu Suomela, ${ }^{1}$ Rebecca Schmidt, ${ }^{1,2,3}$ and Tapio Ala-Nissila ${ }^{1,4}$ \\ ${ }^{1}$ COMP Center of Excellence, Department of Applied Physics, Aalto University, P.O. Box 11000, FI-00076 Aalto, Finland \\ ${ }^{2}$ Turku Centre for Quantum Physics, Department of Physics and Astronomy, University of Turku, FIN-20014 Turku, Finland \\ ${ }^{3}$ Center for Quantum Engineering, Department of Applied Physics, Aalto University School of Science, \\ P.O. Box 11000, FIN-00076 Aalto, Finland \\ ${ }^{4}$ Department of Physics, Box 1843, Brown University, Providence, Rhode Island 02912-1843, USA
}

(Received 8 December 2016; published 21 February 2017)

\begin{abstract}
We study non-Markovian effects present in a driven qubit coupled to a finite environment using a recently proposed model developed in the context of calorimetric measurements of open quantum systems. To quantify the degree of non-Markovianity we use the Breuer-Laine-Piilo (BLP) measure [H.-P. Breuer, Phys. Rev. Lett. 103, 210401 (2009)]. We show that information backflow only occurs in the case of driving, in which case we investigate the dependence of memory effects on the environment size, driving amplitude, and coupling to the environment. We show that the degree of non-Markovianity strongly depends on the ratio between the driving amplitude and the coupling strength. We also show that the degree of non-Markovianity does not decrease monotonically as a function of the environment size.
\end{abstract}

DOI: 10.1103/PhysRevA.95.022120

\section{INTRODUCTION}

While the Markovian description is often a very good approximation of an open system's evolution in quantum optics [1], the underlying assumptions, such as a weak system-environment coupling and a memoryless, i.e., infinite, and not too cold environment, can be easily violated in condensed matter systems [2-4]. For such systems a detailed understanding of the memory effects is desirable. The study of non-Markovianity in open quantum systems has become a subject of broad interest in recent years due to the rapid development in quantum information [5,6] and in quantum thermodynamics [7-21]. There is a great variety of nonMarkovianity measures for open quantum systems proposed in the literature [22-30], which are not identical. Some of them, such as the RHP (Rivas-Huelga-Plenio [24]) measure, are based on the nondivisibility of the dynamical map, while other quantifiers focus on the information backflow, such as the BLP (Breuer-Laine-Piilo [22]), which is based on the evolution of the distinguishability of quantum states. The BLP measure is commonly used, as its operational definition allows for a clear physical interpretation of information backflow.

Non-Markovian dynamics is commonly associated with a strong coupling between the system and the environment or parts of the environment [31]. However, the dynamics can become non-Markovian even in the weak coupling regime if the environment is either very cold [20] or finite [21,32-34]. This is especially important in stochastic thermodynamics of open quantum systems, where non-Markovianity plays a crucial role in the detection of heat and work. In stochastic thermodynamics, direct detection of work and heat requires that the heat exchange between the system and the environment leaves detectable traces to the environment. One proposed measurement scheme to do this is the calorimetric detection of the immediate environment $[10,35]$.

\footnotetext{
*rui.ferreirasampaio@aalto.fi
}

In the calorimetric measurement scheme, heat exchange between the system and the environment is obtained by monitoring the environment's energy or effective temperature. However, in order to witness the changes in the environment's state, the environment has to be finite, in contrast to an infinitely large or memoryless environment required for justifying the Markovian approach. For this reason, the calorimetric setup cannot be modeled with the standard Lindblad master equation.

In recent articles [11-13], a modified Lindblad-like equation was introduced that takes into account the finite size of the environment suitable for describing the calorimetric measurement. This modified Lindblad-like equation can be seen as a special case of the formal scheme of coupled master equations described in Refs. [36] and [37]. The corresponding finite-environment master equation (FEME) [12] and its stochastic unraveling [11] lead to stochastic trajectories of the system state that depend on their own history via the state of the environment. Thus, from the point of view of the system's degrees of freedom, the trajectories are non-Markovian [38].

In this article, we study the degree of non-Markovianity induced by the finite size of the environment using the BLP measure. We focus on a driven qubit coupled to an environment consisting of two-level systems with the same energy gap. We assume that the environmental degrees of freedom decohere more rapidly than any other time scale such that we can use the FEME. With the BLP measure, we quantify the level of non-Markovianity for different environment sizes, driving strengths, and system-environment coupling strengths. We show that the FEME leads to non-Markovian dynamics according to the BLP measure in the presence of driving. We additionally show that the degree of non-Markovianity does not always decrease monotonically as a function of the environment size.

\section{MODEL}

We focus on a driven qubit (two level system) with Hamiltonian $H_{\mathrm{q}}(t)=\hbar \omega_{0} a^{\dagger} a+\lambda(t)\left(a^{\dagger}+a\right)$, where $a\left(a^{\dagger}\right)$ is 
(a)

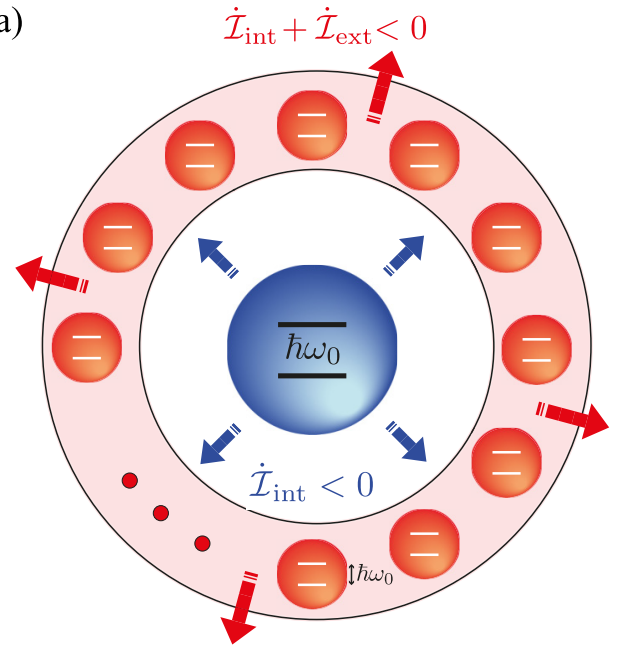

(b)

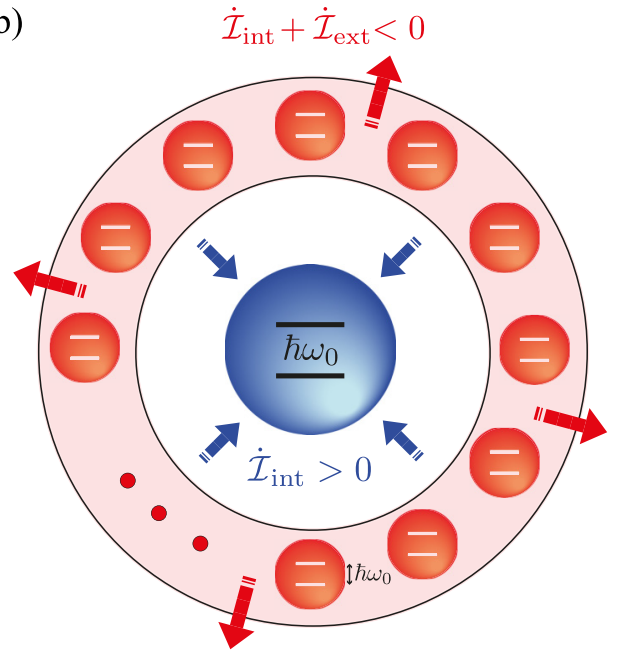

written as

$$
\rho_{\mathrm{QC}}(t)=\sum_{n=0}^{N} \sigma(n, t) \otimes \sigma_{\mathrm{c}}\left(E_{n}\right),
$$

where $n$ denotes the number of excited two-level systems in the calorimeter, $E_{n}=n \hbar \omega_{0}$ denotes the corresponding calorimeter energy, and $\sigma_{c}\left(E_{n}\right)$ is the microcanonical ensemble of calorimeter microstates corresponding to the energy $E_{n}$, i.e., $\sigma_{c}\left(E_{n}\right)=\left[1 / N\left(E_{n}\right)\right] \sum_{k}\left|\Psi_{k}\right\rangle\left\langle\Psi_{k}\right| \delta_{\epsilon_{k}, E_{n}}$, where $\epsilon_{k}$ is the energy of microstate $\left|\Psi_{k}\right\rangle$ and $N\left(E_{n}\right)$ is the number of microstates with energy $E_{n}$. The reduced density matrix is obtained by tracing over all the calorimeter (c) degrees of freedom:

$$
\sigma(t)=\operatorname{Tr}_{\mathrm{c}}\left\{\rho_{\mathrm{QC}}(t)\right\}=\sum_{n=0}^{N} \sigma(n, t) .
$$

Treating the drive and qubit-calorimeter coupling perturbatively, it can be shown [12] that the evolution of $\sigma(n, t)$ obeys a FEME:

$$
\begin{aligned}
\dot{\sigma}(n, t)= & \frac{i}{\hbar}\left[\sigma(n, t), H_{\mathrm{q}}(t)\right]-\frac{\Gamma_{\uparrow}(n)}{2}\left\{\sigma(n, t), a a^{\dagger}\right\} \\
& +\Gamma_{\downarrow}(n-1) a \sigma(n-1, t) a^{\dagger} \\
& +\Gamma_{\uparrow}(n+1) a^{\dagger} \sigma(n+1, t) a \\
& -\frac{\Gamma_{\downarrow}(n)}{2}\left\{\sigma(n, t), a^{\dagger} a\right\},
\end{aligned}
$$

where $\Gamma_{\uparrow}(n)$ and $\Gamma_{\downarrow}(n)$ are the transition rates associated with the Lindblad operators $a^{\dagger}$ and $a$, respectively. The transition rates depend on the calorimeter energy $E_{n}$ and are expressed as

$$
\begin{gathered}
\Gamma_{\downarrow}(n)=g(1-n / N), \\
\Gamma_{\uparrow}(n)=g n / N,
\end{gathered}
$$

where $g$ is a tunable parameter representing the coupling strength between the system and the calorimeter. Because these transition rates depend on the energy of the calorimeter, the evolution of the qubit depends on its history.

In general, Eq. (3) produces a divisible map only for the set of $\sigma(n, t)$, and not for the reduced density matrix of the qubit. Thus, the dynamics of the latter can be non-Markovian, according to the divisibility definition of Markovianity [5,24]. The evolution of the qubit-calorimeter composite [see Eq. (1)] is specified by the set of coupled master equations in Eq. (3), which contain more information than the knowledge of the reduced density matrix of the qubit. Thus, in general, knowledge of $\sigma(0)$ is not sufficient to specify the evolution of the set $\{\sigma(n, t)\}$. In Ref. [12] the Lindblad master equation for the qubit-calorimeter composite is explicitly given, together with the decomposition in terms of coupled master equations for the set $\{\sigma(n, t)\}$. It is important to note that $\sigma(n, t)$ cannot be interpreted as a reduced density matrix. Rather, they are the block components of the qubit-calorimeter density matrix in Eq. (1) and its trace represents the probability $p_{n}(t)$ of finding the calorimeter with a given energy $E_{n}$ as a function of time. Although the map at the level of the set $\{\sigma(n, t)\}$ is Markovian, when the trace operation is performed to obtain the map at 
the level of the qubit reduced density matrix, information is lost and the map becomes, in general, non-Markovian. This relation between the set of matrices $\{\sigma(n, t)\}$ and the reduced density matrix $\sigma(t)$ has been introduced at a formal level in Ref. [36] and discussed further in Refs. [37,40].

In order to quantitatively study non-Markovianity and the degree of it generated by Eqs. (2) and (3), we use the BLP measure, which focuses on the distinguishability of quantum states. One reason to choose the BLP measure is that if the dynamics are non-Markovian according to the BLP measure, then the dynamics are non-Markovian also according to other measures such as the nondivisibility and the semigroup properties [5]. Formally, the BLP measure $\mathcal{N}(\Phi)$ is given by $[6,22]$

$$
\mathcal{N}(\Phi)=\max _{\sigma^{1,2}} \int_{\dot{\mathcal{I}}(t)>0} d t \dot{\mathcal{I}}(t)
$$

where $\mathcal{I}(t) \equiv \mathcal{I}\left(\Phi_{t} \sigma^{1}, \Phi_{t} \sigma^{2}\right)=\left\|\Phi_{t}\left(\sigma^{1}\right)-\Phi_{t}\left(\sigma^{2}\right)\right\| / 2$ is the trace distance between $\Phi_{t} \sigma^{1,2}$ as a function of time, and $\Phi_{t}$ is a linear map of the reduced density matrix associated with the formal solution of the set of Eq. (3), i.e., $\sigma(t)=\Phi_{t} \sigma(0)$. The integral is taken over all the regions where the trace distance rate $\dot{\mathcal{I}}(t)$ is positive and the maximization is performed over all possible pairs of initial states for the reduced density matrices. It is important to note that the map $\Phi_{t}$ acts on the reduced density matrix and not on the set $\{\sigma(n, t)\}$. Since in general this map cannot be calculated analytically for this system, one has to numerically solve the set of coupled master equations in Eq. (3) and then perform the trace operation as described in Eq. (2). If the initial qubit-calorimeter state is a product state, such that $\rho_{\mathrm{QC}}(0)=\sigma(0) \otimes \sigma_{\mathrm{C}}(0)$ and we fix $\sigma_{\mathrm{C}}(0)$, the map of the reduced density state $\sigma(t)$ can be reconstructed. It is crucial that $\sigma_{\mathrm{C}}(0)$ is fixed for all solutions since otherwise there would not be a transformation which assigns a unique $\sigma(t)$ to each $\sigma(0)$. This is required to evaluate non-Markovianity measures since a well-defined map for the reduced density matrix of the system is needed. For the particular case of the qubit, it has been shown that the optimal pair must be pure orthogonal states [41]. Therefore, the initial condition $\sigma^{1}-\sigma^{2} \equiv \tilde{\sigma}_{0}$ can be written as

$$
\tilde{\sigma}_{0}=\left(\begin{array}{cc}
\cos \theta & e^{i \phi} \sin \theta \\
e^{-i \phi} \sin \theta & -\cos \theta
\end{array}\right),
$$

where $\theta$ and $\phi$ are the usual Bloch sphere angles. With these simplifications, the maximization is taken over $\theta \in[0, \pi[$ and $\phi \in[0, \pi[$. In all of the following results, the calorimeter starts from canonical equilibrium at inverse temperature $\beta$ such that the initial condition for the set $\{\tilde{\sigma}(n, 0)\} \equiv\left\{\sigma^{1}(n, 0)-\right.$ $\left.\sigma^{2}(n, 0)\right\}$ is given by

$$
\tilde{\sigma}(n, 0)=p_{n}(0) \tilde{\sigma}_{0},
$$

with $p_{n}(0)=\left(\begin{array}{l}N \\ n\end{array}\right) \exp \left[-\beta\left(E_{n}-F\right)\right]$, where $F$ is the free energy. Furthermore, we use a sinusoidal driving protocol $\lambda(t)=\lambda_{0} \sin \left(\omega_{0} t\right)$ resonant with the qubit [see the Appendix for details on the evaluation of $\mathcal{N}(\Phi)]$.

\section{RESULTS}

\section{A. Trace distance behavior}

We start by discussing the main characteristics of $\dot{\mathcal{I}}(t)$ for the system studied. Figure 2 shows a typical example for a particular set of parameters (see the figure caption for details). For consistency, we employ the notation in Ref. [6], denoting $\mathcal{I}_{\text {int }} \equiv \mathcal{I}$ and $\mathcal{I}_{\text {ext }}=D\left(\rho_{\mathrm{QC}}^{1}(t), \rho_{\mathrm{QC}}^{2}(t)\right)-\mathcal{I}_{\text {int }}$, where $D\left(\rho_{\mathrm{QC}}^{1}(t), \rho_{\mathrm{QC}}^{2}(t)\right)$ is the trace distance between $\rho_{\mathrm{QC}}^{1}$ and $\rho_{\mathrm{QC}}^{2}$.

The notation emphasizes the connection to the information theoretic interpretation of the BLP measure. $\mathcal{I}_{\text {int }}(t)$ represents the distinguishability of the qubit reduced state, while $\mathcal{I}_{\text {ext }}(t)$ represents the distinguishability of the total system states minus the distinguishability of the qubit states. For brevity, we refer to $\mathcal{I}_{\text {int }}(t)$ and $\mathcal{I}_{\text {ext }}(t)$ as the information in the qubit and calorimeter, respectively. We should emphasize at this point that $\mathcal{I}_{\text {int }}+\mathcal{I}_{\text {ext }}$ is not a conserved quantity since some of the information in the qubit-calorimeter system is constantly lost, in accordance with the Markovian dissipative dynamics of $\rho_{\mathrm{QC}}(t)$ given by the set of coupled master equations in Eq. (3). Suppose that we explicitly take into account the outer environment responsible for this loss of information. One would define $\mathcal{I}_{\mathrm{QC}} \equiv D\left(\rho_{\mathrm{QC}}^{1}(t), \rho_{\mathrm{QC}}^{2}(t)\right)$ and $\mathcal{I}_{\mathrm{E}} \equiv D\left(\rho_{\mathrm{QCE}}^{1}(t), \rho_{\mathrm{QCE}}^{2}(t)\right)-\mathcal{I}_{\mathrm{QC}}$, where $\rho_{\mathrm{QCE}}^{1,2}(t)$ stands for the density matrices of the tripartite system, such that $\mathcal{I}_{\mathrm{QC}}+\mathcal{I}_{\mathrm{E}}=$ const. However, we are interested in the distinguishability of the reduced density matrices of the system of interest, the qubit in this case, which is contained in the quantity $\mathcal{I}_{\text {int }} \equiv D\left(\sigma^{1}(t), \sigma^{2}(t)\right)$. What is left is what we have called the

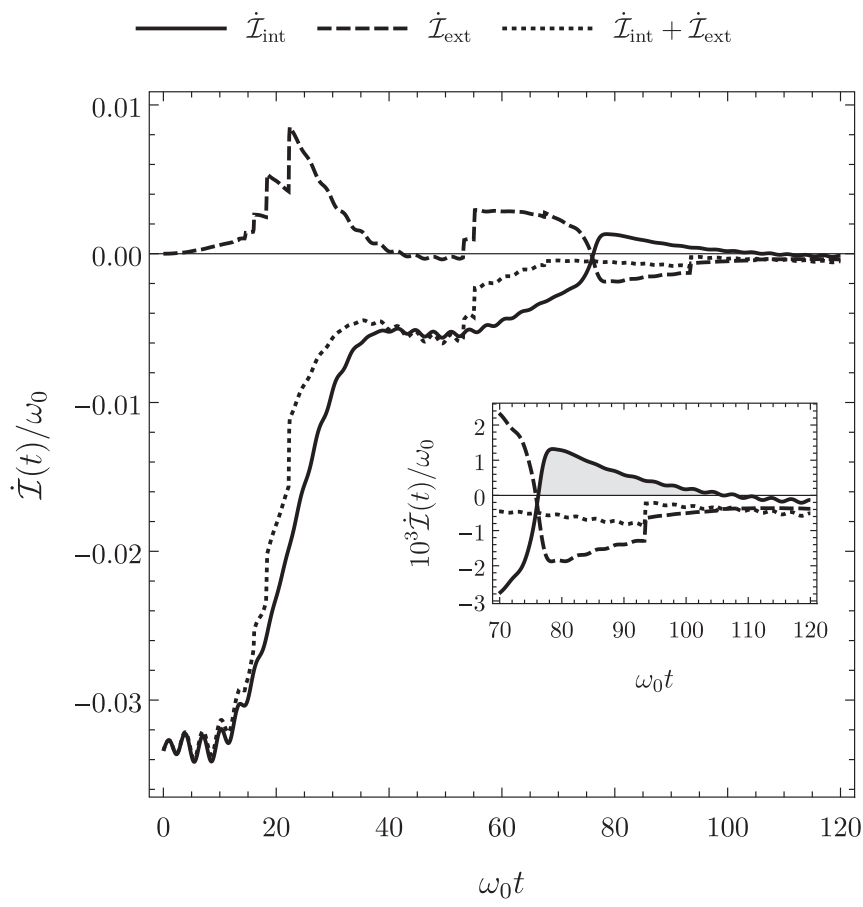

FIG. 2. Rate of change of the information in the qubit, $\dot{\mathcal{I}}_{\text {int }}$, the information in the calorimeter, $\dot{\mathcal{I}}_{\text {ext }}$, and the total information, $\dot{\mathcal{I}}_{\text {int }}+\dot{\mathcal{I}}_{\text {ext }}$, as a function of time. Inset: Region of the evolution that contributes the BLP measure in Eq. (6). Parameters used are $\beta \hbar \omega_{0}=2, \lambda_{0} / \hbar \omega_{0}=0.08, g / \hbar \omega_{0}=0.066, N=20, \theta=1.69$, and $\phi=0$. 
"information in the calorimeter", $\mathcal{I}_{\text {ext }} \equiv D\left(\rho_{\mathrm{QC}}^{1}(t), \rho_{\mathrm{QC}}^{2}(t)\right)-$ $\mathcal{I}_{\text {int }}$, such that $\mathcal{I}_{\text {int }}+\mathcal{I}_{\text {ext }}+\mathcal{I}_{\mathrm{E}}=$ const. In terms of flow of information, the loss (gain) of information in the qubit may not correspond to the gain (loss) of information in the calorimeter since $\dot{\mathcal{I}}_{\text {int }}=-\dot{\mathcal{I}}_{\text {ext }}-\dot{\mathcal{I}}_{\mathrm{E}}$. Indeed, from Fig. 2 it is clear that $\mathcal{I}_{\text {int }}+\mathcal{I}_{\text {ext }}$ is not conserved and its rate of change is always negative in sign.

Other features that draw immediate attention are the discontinuities of $\dot{\mathcal{I}}_{\text {ext }}(t)$. They arise from the fact that the eigenvalues of the difference between $\rho_{\mathrm{QC}}^{1}$ and $\rho_{\mathrm{QC}}^{2}$ can cross between positive and negative values. By definition, the trace distance is not differentiable at these points, which leads to the discontinuities in Fig. 2. For the qubit trace distance, discontinuities are not present because one of the eigenvalues of $\Phi_{t} \tilde{\sigma}_{0}$ is always positive and the other always negative.

Contrary to other systems in which non-Markovianity has been investigated $[6,20,22]$, the trace distance rate starts from negative values. In those works, the time-dependent transition rates are continuous at time $t=0$ and start from 0 . However, in our case, the transition rates [Eqs. (4) and (5)] are positive and time independent, which leads to discontinuous transition rates at time $t=0$ [42].

It is important to note that non-Markovianity in this system is induced by the driving. If no driving is present, the set of Eqs. (3) can be solved exactly and the trace distance is given by

$$
\mathcal{I}(t)=\sqrt{f(t)^{2} \cos ^{2} \theta+g(t)^{2} \sin ^{2} \theta},
$$

where $\quad f(t)=(N \exp [-g(1+1 / N) t]+1) /(N+1) \quad$ and $g(t)=\exp (-g t / 2)$ and we have explicitly assumed the initial condition in Eq. (8). Since $f(t)$ and $g(t)$ are monotonically decreasing functions of $t, \mathcal{N}(\Phi)=0$. Thus, the dynamics are Markovian according to the BLP measure in the case of no driving. However, it should be noted that even in this case, Eq. (3) generally produces dynamics that are nondivisible for the reduced density matrix and thus the dynamics become non-Markovian according to measures based on the nondivisibility, such as the RHP measure.

The inset in Fig. 2 highlights the part of the evolution that contributes to the BLP measure. Pairs that maximize the BLP measure present sharp transitions from negative to positive values of $\dot{\mathcal{I}}(t)$. At this point information starts flowing back into the qubit.

The BLP measure considers only maximization over initial state pairs. From the experimental point of view it is interesting to consider how the parameters of the system affect $\mathcal{N}(\Phi)$. For our system, there are four such parameters: the calorimeter size $N$, the coupling strength between the qubit and the calorimeter $g$, the driving strength $\lambda_{0}$, and the inverse temperature $\beta$. In the next section we examine how $\mathcal{N}(\Phi)$ depends on the first three parameters. We limit ourselves to the case of low temperatures such that $\beta \hbar \omega_{0}>1$ since the calorimetric detection works in this regime. In all of the following results, the temperature is fixed according to $\beta \hbar \omega_{0}=2$.

\section{B. Influence of the system parameters on $\mathcal{N}(\Phi)$}

To show how the degree of non-Markovianity depends on the system parameters, we plot $\mathcal{N}(\Phi)$ in Fig. 3 as a function of $\lambda_{0}$ and $g$, for three calorimeter sizes, $N=5$ (top), 50
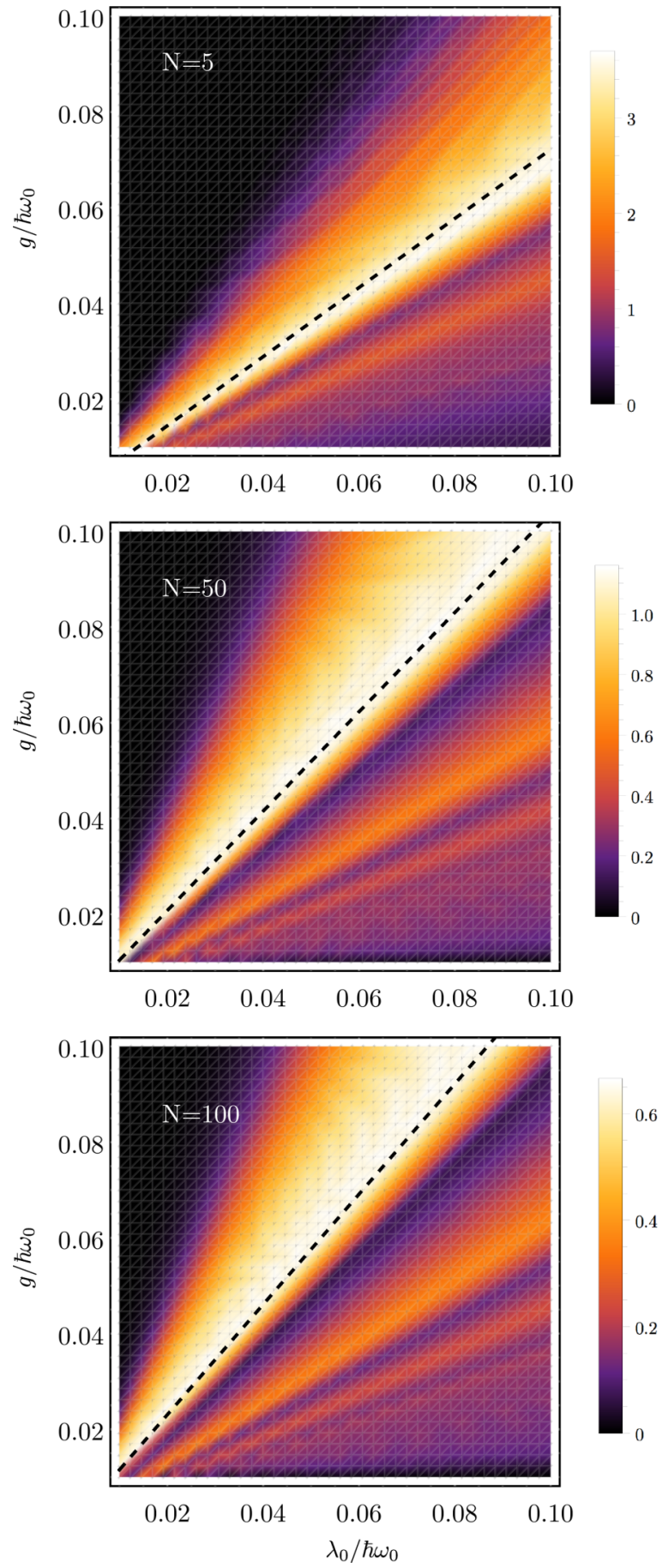

FIG. 3. BLP measure $\left[10^{2} \mathcal{N}(\Phi)\right.$, color scale $]$ as a function of the driving strength $\lambda_{0}$ and the coupling constant $g$ in a grid of $40 \times 40$ points for 5 (top), 50 (middle), and 100 (bottom) two-level systems in the calorimeter. The dashed line indicates the line of constant maximum $\mathcal{N}_{\max }(N)$, given by $\lambda_{0} / g=a_{N}$.

(middle), and 100 (bottom). A non-Markovian structure emerges characterized by "rays" of constant $\mathcal{N}(\Phi)$. An interesting aspect is the oscillatory nature of this structure. It shows that one cannot deduce that a smaller calorimeter will necessarily always induce a higher degree of nonMarkovianity compared to a larger calorimeter. In fact, for fixed $\lambda_{0}$ and $g$ we can go from Markovian to non-Markovian 


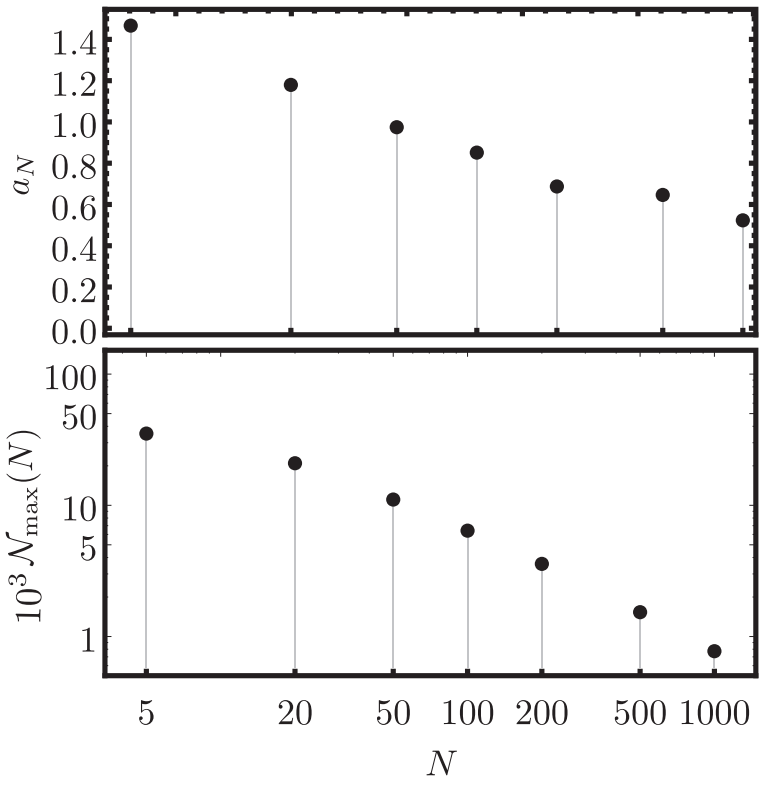

FIG. 4. Log-linear plot of the ratio $a_{N}$ and $\log -\log$ plot of $\mathcal{N}_{\max }(N)$ as a function of the calorimeter size $N$.

behavior by increasing the calorimeter size. On the other hand, at a fixed calorimeter size, one can choose between Markovian and non-Markovian behavior by tuning the coupling to the environment or the driving strength.

The figure of merit for a particular value of $N$ is the peak value of $\mathcal{N}(\Phi)$, which we label $\mathcal{N}_{\max }(N)$. Depending on the specific application one may be interested in asking, for example, How does $\mathcal{N}(\Phi)$ change for fixed system parameters? How does $\mathcal{N}_{\max }(N)$ change within a certain range of parameters? or How does one assure that non-Markovian effects are mitigated? Here, we focus on how $\mathcal{N}_{\text {max }}(N)$ changes as a function of the calorimeter size.

The peak value $\mathcal{N}_{\max }(N)$ falls in the straight line $\lambda_{0} / g=a_{N}$ (cf. Fig. 3). In Fig. 4, we see that $\mathcal{N}_{\max }(N)$ decreases as the calorimeter size increases and seems to converge to $\mathcal{N}_{\text {max }}(N) \rightarrow 0$ as $N \rightarrow \infty$. This is expected, as the relative energy fluctuations around the calorimeter average energy become 0 for $N \rightarrow \infty$. As the driving time and amplitude are finite, the transition rates become constant, as the changes in the calorimeter energy due to the driving are negligible compared to the initial average energy for $N \rightarrow \infty$. Consequently, in this limit the dynamics of the reduced density matrix converge to a Lindblad master equation. We also note that the value of $\mathcal{N}_{\max }(N)$ is rather small for large sizes of the calorimeter. For $N=10^{3}, \mathcal{N}_{\max }(N) \sim 10^{-3}$. This means that the amount of information recovered is not significant.

Finally, as highlighted in Fig. 2, $\dot{\mathcal{I}}$ exhibits a sharp transition from negative to positive at a given time, denoted $t_{R}$. From the information theoretic or reservoir engineering perspective it is useful to know the first point in time at which information flows back to the system. As in the case of $\mathcal{N}(\Phi), t_{R}$ varies with the parameter used. However, if we focus on the line of $\mathcal{N}_{\text {max }}(N)$, a clear pattern emerges. Figure 5 shows a log-log plot of $t_{R}$ along the line $\lambda_{0} / g=a_{N}$, as a function of $\lambda_{0}$, for the three calorimeter sizes, $N=5,50$, and 100. Remarkably, $t_{R}$ is almost independent of the calorimeter size and we

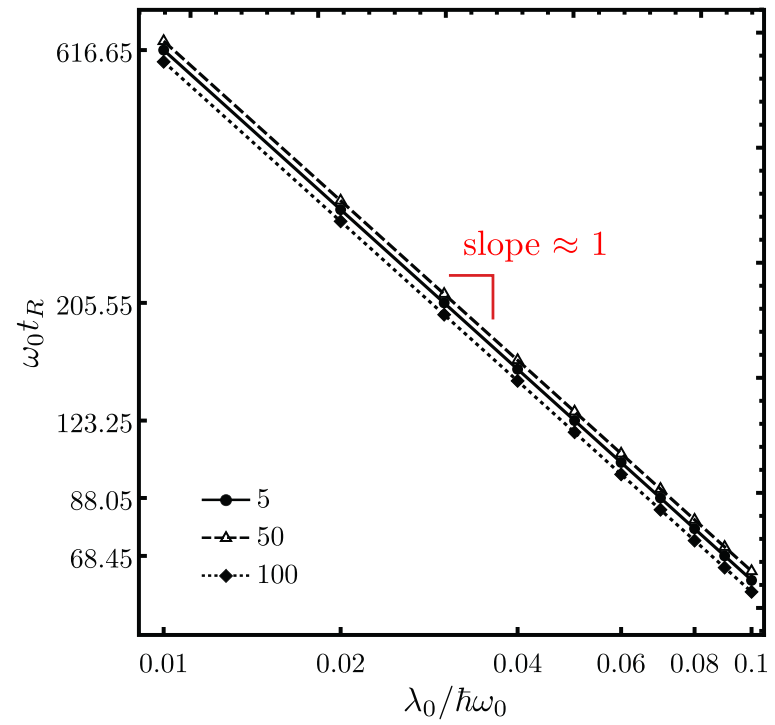

FIG. 5. Log-log plot of $\omega_{0} t_{R}$ along the line of maximum $\mathcal{N}(\Phi)$ $\left(\lambda_{0}=a_{N} g\right)$ for the three calorimeter sizes $N=5,50$, and 100 .

can write

$$
t_{R} \propto \frac{\hbar}{\lambda_{0}} .
$$

Note that this is not true if $\lambda_{0}$ and $g$ are held fixed and the size $N$ is varied. In Fig. 5 and Eq. (10), for a given $\lambda_{0}$ and $N$, the coupling $g$ is implicitly given by $g=\lambda_{0} / a_{N}$. That is, the time at which information starts to flow back into the system can be easily tuned by adjusting the driving amplitude.

\section{SUMMARY AND CONCLUSIONS}

In this article, we have studied the non-Markovianity of the recently proposed FEME, which includes finite-size effects of the environment. We have focused on a driven qubit coupled to an environment consisting of two-level system with the same energy gap as the qubit. We have shown that the FEME produces non-Markovian dynamics according to the BLP measure in the presence of an external drive. This implies that the model produces non-Markovian dynamics also with other definitions of Markovianity based on nondivisibility and semigroup properties [5].

With the BLP measure, we have shown that the degree of non-Markovianity strongly depends on the ratio of the driving amplitude and the qubit-environment coupling strength as witnessed in Fig. 3. Surprisingly, for a fixed value of the driving amplitude and the coupling strength, the degree of nonMarkovianity does not decrease monotonically as a function of the number of two-level systems in the environment. However, if maximized over all possible values of the coupling strength and the drive amplitude, the degree of non-Markovianity tends to decrease exponentially towards 0 as a function of the environment size. For this reason, the amount of recoherence or information recovered is relatively small for very large environments.

We have also investigated the behavior of the time $\left(t=t_{R}\right)$ when the information backflow first occurs. We show that when non-Markovianity is maximized over the coupling strength, $t_{R}$ decreases linearly as a function of the drive amplitude 
for all environment sizes. This means that the occurrence of information backflow can be easily shifted by adjusting the driving field. That is, if non-Markovianity is harmful to the performance of the system, one can shift the occurrence of information backflow to time values larger than the operation time. On the other hand, if one wants to use the information backflow as a resource, one can shift it to occur at a time at which its effect on the system performance is the largest.

\section{ACKNOWLEDGMENTS}

We thank Jyrki Piilo for fruitful discussions. We gratefully acknowledge financial support from the Academy of Finland through project number 287750, the Centers of Excellence Programme (2015-2017) under project numbers 251748 and 284621, and the Centre of Quantum Engineering at Aalto University School of Science. S.S. acknowledges financial support from the Väisälä foundation. The numerical calculations were performed using computer resources of the Aalto University School of Science "Science-IT" project.

\section{APPENDIX: MAXIMIZATION IN THE BLP MEASURE}

To perform the maximization of $\mathcal{N}(\Phi)$ in Eq. (6) in the text, we have to solve the system of equations (3). To this end, we work in the interaction picture with respect to $H_{0} \equiv \hbar \omega_{0} a^{\dagger} a$. Then one has to solve the evolution dictated by the $4(N+1)$ equations,

$$
\begin{aligned}
\dot{\sigma}_{00}^{n}(t)= & i v^{*} \sigma_{01}^{n}(t)-i v \sigma_{10}^{n}(t)-\Gamma_{\uparrow}(n) \sigma_{00}^{n}(t) \\
& +\Gamma_{\downarrow}(n-1) \sigma_{11}^{n-1}(t),
\end{aligned}
$$

$$
\begin{aligned}
\dot{\sigma}_{11}^{n}(t)= & i v \sigma_{10}^{n}(t)-i v^{*} \sigma_{01}^{n}(t)-\Gamma_{\downarrow}(n) \sigma_{11}^{n}(t) \\
& +\Gamma_{\uparrow}(n+1) \sigma_{00}^{n+1}(t), \\
\dot{\sigma}_{01}^{n}(t)= & i v\left(\sigma_{00}^{n}(t)-\sigma_{11}^{n}(t)\right)-\frac{\Gamma_{\Sigma}}{2} \sigma_{01}^{n}(t), \\
\dot{\sigma}_{10}^{n}(t)= & i v^{*}\left(\sigma_{11}^{n}(t)-\sigma_{00}^{n}(t)\right)-\frac{\Gamma_{\Sigma}}{2} \sigma_{10}^{n}(t),
\end{aligned}
$$

where $\Gamma_{\Sigma}=\Gamma_{\uparrow}(n)+\Gamma_{\downarrow}(n)=g, \sigma_{i j}^{n}(t)=\langle i|\sigma(n, t)| j\rangle$, and $v=\lambda_{0} \exp \left[-i \omega_{0} t\right] \sin \left(\omega_{0} t\right)$. From Eq. (2), the trace distance rate $\dot{\mathcal{I}}(t)$ is given by

$$
\dot{\mathcal{I}}(t)=\frac{1}{\mathcal{I}(t)}\left(\sigma_{00}(t) \frac{\mathrm{d} \sigma_{00}(\mathrm{t})}{\mathrm{dt}}+\left|\sigma_{01}(t)\right| \frac{\mathrm{d}\left|\sigma_{01}(\mathrm{t})\right|}{\mathrm{dt}}\right),
$$

where $\sigma_{i j}(t)=\sum_{n} \sigma_{i j}^{n}(t)$. This is then integrated over all regions where $\dot{\mathcal{I}}(t)>0$ for a particular initial condition, given by Eqs. (7) and (8) in the text. In practice, we have to truncate the solution after some time $\tau$. We have used $\omega_{0} \tau=1000 \pi$, after which all the solutions show exponential decay. Finally, for given parameters $\lambda_{0}$ and $g$ we sample half of the Bloch sphere in steps of $0.08 \mathrm{rad}$ in both $\theta$ and $\phi$. The maximum always appears for $\phi=0$ or $\phi=\pi$. The only exception is when the maximum is along $\theta=0$ or $\theta=\pi$, for which the solution is independent of $\phi$. This suggests that the optimal pair lines up with the plane formed by the driving term eigenvectors and the undriven qubit Hamiltonian eigenvectors. To further test this, we change the coupling of the driving to be proportional to the Pauli $\sigma_{y}$ matrix, resulting in the optimal pair being in the $y z$ plane (results not shown). Numerical evidence shows that all solutions share this symmetry and we therefore assume in all the calculations that the optimal pair is in the $x z$ plane of the Bloch sphere by setting $\phi=0$.
[1] H. Breuer and F. Petruccione, The Theory of Open Quantum Systems (Oxford University Press, New York, 2002).

[2] J. P. Pekola, P. Muratore-Ginanneschi, A. Kupiainen, and Y. M. Galperin, Phys. Rev. E 94, 022123 (2016).

[3] J. P. Pekola, Nat. Phys. 11, 118 (2015).

[4] R. Schmidt, M. F. Carusela, J. P. Pekola, S. Suomela, and J. Ankerhold, Phys. Rev. B 91, 224303 (2015).

[5] Á. Rivas, S. F. Huelga, and M. B. Plenio, Rep. Prog. Phys. 77, 094001 (2014).

[6] H.-P. Breuer, E.-M. Laine, J. Piilo, and B. Vacchini, Rev. Mod. Phys. 88, 021002 (2016).

[7] M. Esposito, U. Harbola, and S. Mukamel, Rev. Mod. Phys. 81, 1665 (2009).

[8] M. Campisi, P. Hänggi, and P. Talkner, Rev. Mod. Phys. 83, 771 (2011).

[9] B. Leggio, A. Napoli, H.-P. Breuer, and A. Messina, Phys. Rev. E 87, 032113 (2013).

[10] J. P. Pekola, P. Solinas, A. Shnirman, and D. V. Averin, New J. Phys. 15, 115006 (2013).

[11] S. Suomela, A. Kutvonen, and T. Ala-Nissila, Phys. Rev. E 93, 062106 (2016).
[12] S. Suomela, R. Sampaio, and T. Ala-Nissila, Phys. Rev. E 94, 032138 (2016).

[13] J. P. Pekola, S. Suomela, and Y. M. Galperin, J. Low Temp. Phys. 184, 1015 (2016).

[14] G. Katz and R. Kosloff, Entropy 18, 186 (2016).

[15] B. Bylicka, M. Tukiainen, D. Chruściński, J. Piilo, and S. Maniscalco, Sci. Rep. 627989 (2016).

[16] P. Strasberg, G. Schaller, N. Lambert, and T. Brandes, New J. Phys. 18, 073007 (2016).

[17] D. Newman, F. Mintert, and A. Nazir, arXiv:1609.04035.

[18] A. Kato and Y. Tanimura, J. Chem. Phys. 145, 224105 (2016).

[19] R. S. Whitney, arXiv:1611.00670.

[20] R. Schmidt, S. Maniscalco, and T. Ala-Nissila, Phys. Rev. A 94, 010101 (2016).

[21] R. Uzdin and R. Kosloff, arXiv:1610.02671.

[22] H.-P. Breuer, E.-M. Laine, and J. Piilo, Phys. Rev. Lett. 103, 210401 (2009).

[23] E.-M. Laine, J. Piilo, and H.-P. Breuer, Phys. Rev. A 81, 062115 (2010).

[24] Á. Rivas, S. F. Huelga, and M. B. Plenio, Phys. Rev. Lett. 105, 050403 (2010). 
[25] X.-M. Lu, X. Wang, and C. P. Sun, Phys. Rev. A 82, 042103 (2010).

[26] R. Vasile, S. Maniscalco, M. G. A. Paris, H.-P. Breuer, and J. Piilo, Phys. Rev. A 84, 052118 (2011).

[27] S. Luo, S. Fu, and H. Song, Phys. Rev. A 86, 044101 (2012).

[28] S. Lorenzo, F. Plastina, and M. Paternostro, Phys. Rev. A 88, 020102 (2013).

[29] B. Bylicka, D. Chruściński, and S. Maniscalco, Sci. Rep. 45720 (2014).

[30] C. Addis, B. Bylicka, D. Chruściński, and S. Maniscalco, Phys. Rev. A 90, 052103 (2014).

[31] L. Mazzola, S. Maniscalco, J. Piilo, K.-A. Suominen, and B. M. Garraway, Phys. Rev. A 79, 042302 (2009).

[32] T. J. G. Apollaro, C. Di Franco, F. Plastina, and M. Paternostro, Phys. Rev. A 83, 032103 (2011).

[33] M. Žnidarič, C. Pineda, and I. García-Mata, Phys. Rev. Lett. 107, 080404 (2011).
[34] J. Jeske, J. H. Cole, C. Müller, M. Marthaler, and G. Schön, New J. Phys. 14, 023013 (2012).

[35] K. L. Viisanen, S. Suomela, S. Gasparinetti, O.-P. Saira, J. Ankerhold, and J. P. Pekola, New J. Phys. 17, 55014 (2015).

[36] H.-P. Breuer, Phys. Rev. A 75, 022103 (2007).

[37] H.-P. Breuer, J. Gemmer, and M. Michel, Phys. Rev. E 73, 016139 (2006).

[38] This non-Markovianity clearly differs from the non-Markovian trajectories of the non-Markovian quantum jump model [39], where the evolution of a trajectory does not depend on its own history but only on the collective history of all the trajectories.

[39] J. Piilo, S. Maniscalco, K. Härkönen, and K.-A. Suominen, Phys. Rev. Lett. 100, 180402 (2008).

[40] B. Vacchini, Phys. Rev. A 78, 022112 (2008).

[41] S. Wißmann, A. Karlsson, E.-M. Laine, J. Piilo, and H.-P. Breuer, Phys. Rev. A 86, 062108 (2012).

[42] The same behavior occurs using the Lindblad equation with time-independent transition rates. 